\title{
ANDES

\section{Consentimiento sexual y consumo de alcohol en adolescentes. Un análisis integral}

\author{
Sexual consent and alcohol consumption in adolescents. \\ A comprehensive analysis
}

\author{
Pamela Berta Oyarzún Gomberoff ${ }^{\mathrm{a}, \mathrm{b}}$
}

aCEMERA; Facultad de medicina, Universidad de Chile. Santiago, Chile.

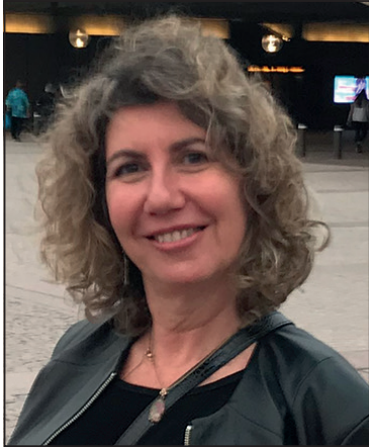

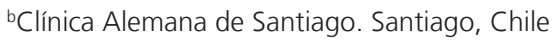

Consentimiento sexual es un concepto que, si bien empezó a gestarse en el siglo XVIII, recién en los años 60-70 comenzó a estructurarse bajo la visión de los derechos de la libertad individual y de la autonomía femenina. Es un concepto que genera debate, se ha escrito mucho, y desde lo jurídico, históricamente ha puesto en evidencia la hegemonía masculina ${ }^{1}$. La discusión se abrió con mayor ímpetu en nuestro país durante el año 2015 a raíz del debate en torno a la legislación sobre la interrupción del embarazo por tres causales donde se hizo evidente que este concepto no estaba para nada claro.

Cuando al escenario de la desinformación en torno al concepto de consentimiento sexual se suma el contexto de consumo de alcohol, las ideas se manifiestan aún más confusas. Es así como hemos sido testigos de declaraciones a la prensa tan poco afortunadas como "Hay mujeres que tienen relaciones porque, a lo mejor, tomaron un traguito de más ies violación también?"i.

Esta falta de claridad, al momento de producirse un acto sexual bajo efectos del alcohol, genera graves consecuencias para los involucrados en el encuentro.

i La tercera. 2015; Available from: https://www.latercera.com/ noticia/lorenzini-hay-mujeres-que-tienen-relaciones-porquea-lo-mejor-tomaron-un-traguito-de-mas-es-violacion-tambien/
Sabemos que el alcohol en el cerebro produce aumento de la impulsividad y disminución del autocontrol, fenómenos que sumados al proceso de maduración propio de la adolescencia pueden ser mas intensos.

La evidencia muestra que, en los adolescentes, el consumo de alcohol se asocia a aumento de las conductas de riesgo, entre las cuales obviamente las del ámbito sexual no son una excepción ${ }^{2}$. Está descrito mayor frecuencia de actividad sexual con desconocidos, más sensación de arrepentimiento y menos uso de condón ${ }^{3,4}$.

En Chile los adolescentes inician consumo de alcohol a edades tan tempranas como a los 13,8 años ${ }^{5}$.

Lo que preocupa más es la forma en que se produce el consumo en este grupo etario, el que con frecuencia lleva a alcanzar rápidamente estados de embriaguez. La comúnmente conocida como borrachera express, o binge drinking definida originalmente por el National Institute on Alcohol Abuse and Alcoholism (NIAAA) el 2004 como ingesta de 5 tragos en hombres y de 4 tragos en mujeres en un lapso de 2 horas, es tan frecuente en nuestro medio, como que el $64 \%$ de los adolescentes que relatan consumo de alcohol el último mes presenta este tipo de conducta ${ }^{5}$. Si bien las cifras de las últimas encuestas Instituto Nacional de la juventud (INJUV)

Correspondencia:

Pamela Berta Oyarzún Gomberoff

pameoyarzun@gmail.com 
del 2018 y Servicio nacional para la Prevención y Rehabilitación del Consumo de Drogas y Alcohol (SENDA) del 2019 muestran una discreta tendencia a la baja del consumo de alcohol en general y del número de tragos por evento, las cifras siguen siendo alarmantes.

Cabe destacar que el 40\% de los jóvenes entre 15 y 19 años refirieron haber consumido alcohol los últimos 12 meses $^{6}$, y que el $29 \%$ de los escolares entre $8^{\circ}$ año de educación básica y $4^{\circ}$ año de educación media declara haber consumido alcohol el último mes ${ }^{7}$, entendiendo que el consumo en esta edad es ilegal.

El tema del acceso al alcohol es algo que como sociedad debemos hacernos cargo. Si bien en la última encuesta se evidencia una mayor dificultad para el acceso al alcohol, esta barrera se ve reflejada en una disminución en la compra directa por los escolares. La encuesta muestra que las formas de compra de alcohol en casi un $40 \%$ están facilitadas por otro adulto ${ }^{8}$. Este resultado deja en evidencia el doble estándar que tenemos sobre el consumo de alcohol en la adolescencia, donde los adultos somos quienes les proporcionamos la oportunidad de atravesar las barreras que supuestamente como sociedad decidimos poner.

$\mathrm{Al}$ cruzar el concepto de consentimiento sexual con la conducta del consumo de alcohol nos enfrentamos a la pregunta de "¿Podemos hablar de consentimiento cuando la persona se encuentra en estado de embriaguez?". La respuesta negativa es categórica. Uno de los problemas a los que se enfrenta es que probablemente el que debe solicitar el consentimiento tampoco se encuentra en condiciones de evaluar si lo está obteniendo, ya que probablemente se encuentra en igual estado y por lo tanto incapaz de realmente ver a su pareja, con poca capacidad para comunicarse y percibir lo que le ocurre a su acompañante y con escaso control de sus impulsos. Claramente su incapacidad no le resta responsabilidad, solo deja en evidencia la necesidad imperiosa de realizar campañas efectivas para disminución del consumo de alcohol en nuestros adolescentes.

Las cifras que arrojan los estudios nacionales sobre consumo de alcohol en adolescentes ponen en evidencia una irresponsabilidad colectiva como sociedad. Cuando hablamos de campañas efectivas nos referimos a cambiar el doble estándar de permisividad del consumo. Debemos ser coherentes con el discurso; No debe haber consumo de alcohol en menores de 18 años. En este sentido la experiencia Islandesa, que muestra una disminución de un 49 a un 5\% en los últimos 20 años, es digna de replicar ${ }^{910}$. Se han implementado programas piloto en algunos municipios, esperamos que esta política se replique en todo nuestro país.

La literatura muestra que un porcentaje importante de las agresiones sexuales en jóvenes universitarios ocurre bajo efectos del alcohol ${ }^{11}$.

Entre las medidas a tomar, es evidente que se re- quiere una modificación al código penal que refleje coherencia con un rechazo absoluto a la violencia sexual.

Actualmente existe un proyecto de ley que pretende modificar el artículo 361 del código penal que define el delito de violación como: "Comete violación el que accede carnalmente, por vía vaginal, anal o bucal, a una persona mayor de catorce años, en alguno de los casos siguientes: $1^{\circ}$ Cuando se usa de fuerza o intimidación. $2^{\circ}$ Cuando la víctima se halla privada de sentido, o cuando se aprovecha su incapacidad para oponerse. $3^{\circ}$ Cuando se abusa de la enajenación o trastorno mental de la víctima". Por: "El acceso carnal ....sin el consentimiento de la víctima, constituye violación y será castigado..." incorporando literalmente el concepto de consentimiento en lugar de violación y especifica que un acto sexual no consentido es sinónimo de violación, este proyecto de ley fue aprobado por unanimidad el 4/7/2019 por la cámara de diputados ${ }^{\text {ii }}$ aún está en trámite en la cámara alta.

Desde la perspectiva de salud, la promoción de cambios conductuales parece prioritaria.

La literatura muestra que:

El consumo de alcohol en adolescentes se asocia a múltiples factores de riesgo comunes con la mayoría de las conductas de riego en la adolescencia ${ }^{12,13}$. Llama la atención como los adolescentes asocian el consumo de alcohol a una serie de mitos en relación a los efectos que esta sustancia puede tener sobre la performance sexual. Es interesante lo que muestra Castaño en su investigación, como los escolares atribuyen a la ingesta de alcohol efectos tales como: facilitar el inicio en la practica sexual $47 \%$, aumentar la excitación $26 \%$, prolongar la relación sexual 20\% y evitar la eyaculación precoz en el $13 \%$. Efectos que mencionan como motivadores para consumir esta sustancia ${ }^{14}$.

En este mismo sentido se hace necesario considerar la percepción que tienen los mismos adolescentes de género masculino sobre la violencia sexual. Pareciera que para muchos, ésta es invisible. Olavarría en un estudio que realizó sobre masculinidades describió que los adolescentes varones no visualizan la violencia sexual como tal: relatan no haber estado involucrados en este tipo de situaciones sin embargo al repasar su primera experiencia sexual en un porcentaje importante se evidencia que fue una violación ${ }^{15}$.

Desde esta perspectiva la educación en sexualidad es de vital importancia. Una educación que incorpore temas de género, de masculinidades, de inclusión, no violencia, no discriminación. La incorporación de programas de prevención de violencia sexual en la adolescencia ha mostrado resultados positivos ${ }^{16}$. Educación que para lograr cambios reales debe ser impartida des-

ii Emol.com - https://www.emol.com/noticias/Nacional/2019/07/ 04/953583/Camara-aprueba-por-unanimidad-el-proyecto-Sinconsentimiento-es-violacion-y-ahora-pasara-al-Senado.htm 
de la educación parvularia, adecuando los contenidos a cada edad y grado de desarrollo de los niños y adolescentes. Es necesario visibilizar y sensibilizar sobre el tema, evitando malos entendidos sobre el significado de "educación en sexualidad" y el alcance que tiene la incorporación de estos temas desde el periodo preescolar. Situación que en nuestro país determinó el rechazo al proyecto de ley para Educación sexual integral (ESI) $)^{\text {iii. }}$.

Resumiendo, parece claro que es imposible hablar de consentimiento sexual cuando se está bajo efectos del alcohol.

Que para prevenir este tipo de situaciones es necesario abordar el tema desde varias perspectivas y tomar conductas concordantes:

1. Incorporar la cultura del consentimiento a través de:

- Concretar las modificaciones al código penal.

- Consolidar una adecuada educación sexual desde etapas muy tempranas para lograr cambios culturales. Cabe señalar que se lograrán mejores resultados si la generación de programas y su implementación es fruto de un trabajo intersectorial que incorpore a salud y a educación ${ }^{17}$ y considerando una participación de los padres y adolescentes en este proceso.

- Comprometer a la prensa y autoridades para la realización de campañas educativas, insertando cultura de consentimiento dirigidas a toda la población.

- Campañas educativas, sanciones concordantes y acompañamiento a víctimas en establecimientos de educación superior.
2. Disminuir el consumo de alcohol en adolescentes:

- A través de campañas complejas que involucren a toda la sociedad. Tolerancia cero al consumo de alcohol en adolescentes. Esto involucra un cambio cultural esencial en cuanto a la percepción del riesgo del consumo de esta sustancia en la adolescencia y a abordar el problema con determinación, responsabilidad y voluntad política de generar los cambios.

Nosotros como profesionales tenemos un rol importante educando a los padres de nuestros pacientes, realizando consejería ${ }^{18}$. Debemos incorporar aspectos de sexualidad, de consumo y de comunicación, debemos "propiciar la educación en el consentimiento sexual como una forma de prevención de la violencia sexual" tal como explicitan las "Orientaciones técnicas en salud sexual para atención primaria” del ministerio de salud del 2018. Debemos promover una comunicación fluida, clara y empática con los hijos, fomentar que los padres generen espacios en que sus hijos sientan confianza para recurrir a ellos cuando lo necesiten. Es necesario entender que la responsabilidad no es de la víctima.

Por último, es importante entender que este tipo de situaciones pueden disminuir, sin embargo, seguirán ocurriendo. La atención a las víctimas a través de redes de apoyo es prioritaria. Parece lamentable que más del $80 \%$ de la víctimas atraviesen por esta situación solas, en silencio, sin que cuenten con la contención necesaria, que no denuncien y no se atrevan a contarlo ni siquiera a sus padres, que 1 de cada 4 víctimas no lo comente con nadie ${ }^{12}$. Muchas veces, además, con sentimientos de vergüenza y de culpa.

iii EMOL [Internet]. 2020; Available from: https://www.emol. com/noticias/Nacional/2020/10/15/1000844/Camara-rechazaEducacion-Sexual-Integral.html

\section{Conflicto de intereses}

La autora declara no tener conflicto de intereses.

\section{Referencias}

1. Pérez- Hernández Y. Consentimiento sexual: Un análisis con perspectiva de género. Rev Mex Sociol. 2016;78(4):74167.

2. Simons JS, Maisto SA, Wray TB. Sexual risk taking among young adult dual alcohol and marijuana users. Addictive Behaviors. 2010;35: 533-6.

3. Fairlie AM, Garcia TA, Lee CM, Lewis MA. Alcohol use and alcohol/ marijuana use during the most recent sexual experience differentially predict characteristics of the sexual experience among sexually active young adult drinkers. Addict Behav 2018;82:105 8 .

4. Ritchwood TD, Ford H, DeCoster J, Sutton M, Lochman JE. Risky sexual behavior and substance use among adolescents: A meta-analysis.-Child. Youth Serv. Rev. 2015;52:74-88.

5. Charme C. Décimo Tercer Estudio Nacional de Drogas en Población Escolar, 2019. PRINCIPALES RESULTADOS [Internet]. Santiago; 2020 Available from: https://www.senda.gob. cl/wp-content/uploads/2021/01/ENPE2019.pdf.

6. INJUV. IX_ENCUESTA_NACIONAL_ DE_LA_JUVENTUD_2018 [Internet].
Santiago; 2018. Available from: http:// www.injuv.gob.cl/9encuesta.

7. Observatorio chileno de drogas. Décimo Segundo Estudio Nacional de Drogas en población escolar, 2017 [Internet]. Santiago; Available from: https://www.senda.gob.cl/wp-content/ uploads/2019/01/ENPE-2017.pdf.

8. Observatorio Chileno de Drogas. Décimo Tercer Estudio Nacional de Drogasen Población Escolar de Chile, 2019, $8^{\circ}$ Básico a $4^{\circ}$ Medio [Internet]. Santiago; 2019. Available from: https:// www.senda.gob.cl/wp-content/ uploads/2021/01/ENPE-2019-30-12-20. pdf. 
9. Kristjansson AL, Mann MJ, Sigfusson J, Thorisdottir IE, Allegrante JP, Sigfusdottir ID. Development and Guiding Principles of the Icelandic Model for Preventing Adolescent Substance Use. Health Promot Pract. 2020;21(1):62-9.

10. Kristjansson AL, Mann MJ, Sigfusson J, Thorisdottir IE, Allegrante JP, Sigfusdottir ID. Implementing the Icelandic Model for Preventing Adolescent Substance Use. Health Promot Pract. 2020;21(1):70-9.

11. Lehrer JA, Lehrer VL, Lehrer EL, Oyarzún PB. Prevalence of and risk factors for sexual victimization in college women in Chile. Int Fam Plan Perspect. 2007;33(04):168-75.

12. Substance use disorder in adolescents: Epidemiology, pathogenesis, clinical manifestations and consequences, course, assessment, and diagnosisUpToDate [Internet]. [cited 2020 Jul 16]. Available from: https://www. uptodate.com/contents/substance-usedisorder-in-adolescents-epidemiologypathogenesis-clinical-manifestations-andconsequences-course-assessment-anddiagnosis?csi=be402006-614a-456d-8cf6a84fd67420c4\&source $=$ contentShare.

13. SENDA. Hombres con Consumo Problemático de Drogas. Tratamiento con Perspectiva de Género. SENDA. Mora M, editor. Santiago; 2016.

14. Castaño GA, Morales SA, Arango OE, Montoya MC y Rodríguez A. Adolescencia, sexo y consumo de drogas una guía para la prevención [Internet]. FUNLAM. Medellín: Fundación Universitaria Luis Amigó; 2013. [cited
31/05/2021]. Available from:https:// www.funlam.edu.co/uploads/ fondoeditorial/166_Adolescencia_,_ sexo_y_consumo_de_drogas.pdf

15. Olavarría J. Hombres identidades y violencia de genero. Rev la Acad Acad humanismo Cris 2001;6:101-27.

16. McNaughton Reyes HL, Graham LM, Chen MS, Baron D, Gibbs A, Groves AK, et al. Adolescent dating violence prevention programmes: a global systematic review of evaluation studies. Lancet Child Adolesc Health;2020;4642(3):1-10.

17. Obach A, Sadler M, Jofré N. Salud sexual y reproductiva del adolescente en Chile. Rev Salud Publica. 2017;19(6):848-54.

18. Millán Klüsse T. Adolescent counseling. Rev Chil Pediatr. 2020;91(1):10-8. 